\title{
Two mathematical models for the correction of carbohydrate and protein interference in the determination of uronic acids by the $m$-hydroxydiphenyl method
}

\author{
Miguel Anxo Murado', José Antonio Vázquez, María Ignacia Montemayor, \\ Marta López Cabo and María del Pilar González
}

Instituto de Investigacións Mariñas (CSIC), r/Eduardo Cabello 6, Vigo-36208, Galicia, Spain

The most common method in the routine determination of uronic acids, the $m$-hydroxydiphenyl reaction, recently adapted to rapid microplate analysis, has as a main inconvenience, in any one of their modalities, interferences due to the frequent presence of proteins and neutral carbohydrates in the samples. Corresponding corrections in the literature are unsatisfactory when applied to complex matrices, and further adaptation to the microplate analysis is not free from additional problems. With particular reference to hyaluronic acid, the interactions between the principal reactants and the interfering materials are studied kinetically under realistic conditions, and simple mathematical models are proposed which satisfactorily describe the experimental results and allow adequate corrections to be made.

\section{Introduction}

Hyaluronic acid (HA) is a heteropolysaccharide of high molecular mass $\left(10^{4}-8 \times 10^{6} \mathrm{Da}\right)$, the repetitive unit of which is the disaccharide consisting of $\mathrm{N}$-acetylglucosamine and glucuronic acid. It is present in many animal tissues (skin, cartilage, umbilical cord, combs (fleshy bird crests), sinovial fluid and vitreous humour), as well as in the cell wall of bacteria such as Streptococcus zooepidemicus. HA has numerous and increasing applications in clinical and cosmetic fields, including intra-ocular surgery, treatment of arthritis, loss of vesical elasticity, reinstatement of sinovial fluid in articulations and major burns. It is also used in plastic surgery and in topical preparations to restore the flexibility of the skin. As a consequence of these applications, requiring a high degree of purification, HA has become a very expensive product, and interest in obtaining it from natural sources and by means of fermentation has increased greatly over the last decade. Purity control along the production process, as well as precision of the clinical analyses (in cases such as the loss of HA in urine or damage to the sinovial fluid), require methods of $\mathrm{HA}$ determination able to correct the most frequent sources of interference (proteins, carbo- hydrates) in the biological matrices usually examined in this respect.

The analytical determination of HA presently relies on a large variety of resources based on techniques such as HPLC [I], immunoassay [2] or electrophoresis [3]. These techniques are suitable for specific aims, such as determining the distribution of molecular masses in the matrices of origin. For general purposes, however, it is important to have rapid and robust methods, not very sensitive to possible interference, and directly applicable to crude or simply pretreated matrices.

Within this second group of methods, the most widely used is based on hydrolysis of the $\mathrm{HA}$ at $100^{\circ} \mathrm{C}$ with concentrated sulphuric acid, followed by reaction of the uronic acid (glucuronic acid) formed with m-hydroxydiphenyl at $22^{\circ} \mathrm{C}$ [4]. Recently adapted to facilitate the assay on microtitre plates [5], the greatest inconveniences of this method are attributed to interference from the high concentrations of proteins and carbohydrates in the samples. In this respect, simply additive corrections, or measuring the difference in absorbance before and after addition of $m$-hydroxydiphenyl (as suggested by Blumenkrantz and Asboe-Hansen [4]), are unsatisfactory. On the other hand, although the abovementioned modification facilitates the use of microplates, diverse additional complications are, in this manner, introduced in the reaction.

Other studies on the same analytical basis have indicated improvement in sensitivity brought about by reducing the temperature of the treatment with sulphuric acid at $80^{\circ} \mathrm{C}$ [6], or by using a treatment with sulphamic acid/potassium sulphamate before heating $\left(\right.$ at $100^{\circ} \mathrm{C}$ ) with sulphuric acid [7]. However, we have found that the stage at $80^{\circ} \mathrm{C}$ increases the variability of the results, and that the sulphamate treatment is delicate, since its effect depends on the relationship between $\mathrm{HA}$ and neutral sugars in the sample. Finally, the automatic corrections (based on

\footnotetext{
Key words: glucose and protein interference, hyaluronic acid $(\mathrm{HA})$,

m-hydroxydiphenyl method, kinetic model, uronic acids.

Abbreviation used: HA, hyaluronic acid.

To whom correspondence should be addressed (email recicla@iim.csic.es).
} 
polynomial fits and incorporated in the software of some instruments) are often very uncertain. It is difficult, therefore, to avoid the conclusion that an appropriate correction of those interferences requires knowledge of the sample concentrations of proteins and glucose.

In the present study the interference of proteins and glucose in the m-hydroxydiphenyl method is studied, as well as the problems derived from its adaptation to the microplate method. Two mathematical models are proposed which adequately describe the interferences and permit satisfactory corrections.

\section{Materials and methods}

With the exception of the HA, all reactants were of analytical quality (Sigma or Merck). HA was obtained from swordfish (Xiphias gladius) and blue-shark (Prionace glauca) vitreous humour, free of neutral carbohydrates, with a protein content of $8 \mu \mathrm{g} / \mathrm{mg}$ of HA and a molecular mass of approx. $2000 \mathrm{kDa}$. The acid was isolated by the procedure of Cullis-Hill [8] combined with diafiltration in a Minitan cell (Millipore, Watford, Herts., U.K.) and omitting acetic acid in the alcoholic precipitation. Proteins were determined by the method of Lowry et al. [9].

\section{Sample pre-treatment and analytical method}

The preparation of crude biological materials was based on a simplified version of the purification method of Rodén et al. [10], substituting the fractionated alcoholic precipitation for a selective redissolution of the sediment obtained by exhaustive precipitation. $\mathrm{NaCl}(0.22 \mathrm{~g})$ was dissolved in $2 \mathrm{ml}$ of vitreous humour, and $4 \mathrm{ml}$ of $100 \%(\mathrm{v} / \mathrm{v})$ ethanol were slowly added with vortex-mixing. The mixture was incubated for $\mathrm{I} \mathrm{h}$ at $4{ }^{\circ} \mathrm{C}$, centrifuged for $15 \mathrm{~min}$ at $3000 \mathrm{~g}$, and the supernatant was discarded. The sediment was then resuspended in $1.25 \mathrm{ml}$ of water/ethanol $(\mathrm{I}: 0.75, \mathrm{v} / \mathrm{v})$, centrifuged again, and the supernatant collected. The sediment was washed with $0.75 \mathrm{ml}$ of the same water/ ethanol solution and discarded, and the supernatants were then combined (extract). The recovery of the method was verified using samples of swordfish vitreous humour supplemented with purified $\mathrm{HA}$, and was always $>97 \%$.

The basic analytical assay was a slight modification of the method of Blumenkrantz and Asboe-Hansen [4], in which the borate concentration was reduced to the level where an improvement in sensibility was undetected. The reactants used were: (A) $\mathrm{Na}_{2} \mathrm{~B}_{4} \mathrm{O}_{7}\left(\mathrm{l} \mathrm{mmol} \cdot \mathrm{I}^{-1}\right.$ in sulphuric acid; $96 \%$, $\mathrm{w} / \mathrm{w})$, and (B) $m$-hydroxydiphenyl $\left(\mathrm{l} \mathrm{mg} \cdot \mathrm{ml}^{-1}\right)$ in $\mathrm{NaOH}$ $\left(0.125 \mathrm{~mol} \cdot \mathrm{I}^{-1}\right)$. The reaction was carried out by combining $0.5 \mathrm{ml}$ of sample and $3.4 \mathrm{ml}$ of reagent $A$ by vortex-mixing, incubating the sample for $5 \mathrm{~min}$ in a boiling-water bath, followed by immediate cooling in a $22^{\circ} \mathrm{C}$ bath. Thereafter, $100 \mu \mathrm{l}$ of reagent $\mathrm{B}$ were added with further vortex-mixing. After $15 \mathrm{~min}$ the absorbance was measured at $520 \mathrm{~nm}$ against a blank consisting of $0.5 \mathrm{ml}$ of distilled water. Calibration was carried out with serial dilutions of glucuronic acid $\left(10-100 \mu \mathrm{g} \cdot \mathrm{ml}^{-1}\right)$. When neutral carbohydrates were present in the sample, the absorbance was also measured at $520 \mathrm{~nm}$ immediately before adding reagent $B$.

\section{Experimental designs}

The experimental designs used to study the effects of the adaptation [5] to the microtitre-plate assay, as well as for determination of the separate effects of proteins and carbohydrates on the determination of glucuronic acid, are described in the Results section. The possibility of joint, nonadditive effects of both interfering materials was investigated by means of a statistical rotatable design $[11,12]$, on the same experimental domain used for the separate effects and five replicates in the centre. The statistical significance of the coefficients was verified by means of the Student's $t$ test $(\alpha=0.05)$, and model consistency by means of the Fisher $F$ test $(\alpha=0.05)$ applied to the following mean square ratios: total error/experimental error and lack of fitting/experimental error.

\section{Results and discussion}

\section{Effects of the tetraborate ion and DMSO (microtitre-plate assay)}

An increase in sensitivity of the $m$-hydroxydiphenyl reaction with tetraborate with some uronic acids (glucuronic acid among others) is well established [4]. However, when we used the method described above and in the specified serial dilution, our results indicate that the effect does not increase above $0.68 \mathrm{mmol} \cdot \mathrm{I}^{-1}$ tetraborate in the final solution $\left(0.80 \mathrm{mmol} \cdot \mathrm{I}^{-1}\right.$ in reagent $\left.A\right)$.

In modifying the method to the microtitre-plate test [5], the alkaline solution replaced sulphuric acid as the vehicle for $m$-hydroxydiphenyl. This requires DMSO as a dispersant, since $m$-hydroxydiphenyl is relatively insoluble in this medium. However, under adequate solubility conditions, the authors found that the sensitivity of the reaction decreased, and thus the proportions of the reactant and tetraborate were increased. Although the results do not absolutely confirm it, they suggest an undesirable interaction between the tetraborate and DMSO.

To verify this hypothesis, the response of four series of dilutions of glucuronic acid were studied. Employing the methods presented here, two concentrations of borate were combined $\left(0.85\right.$ and $102 \mathrm{mmol} \cdot \mathrm{I}^{-1}$ in the reaction mixture) in the absence and presence $[11.4 \%(\mathrm{v} / \mathrm{v})$ in the 

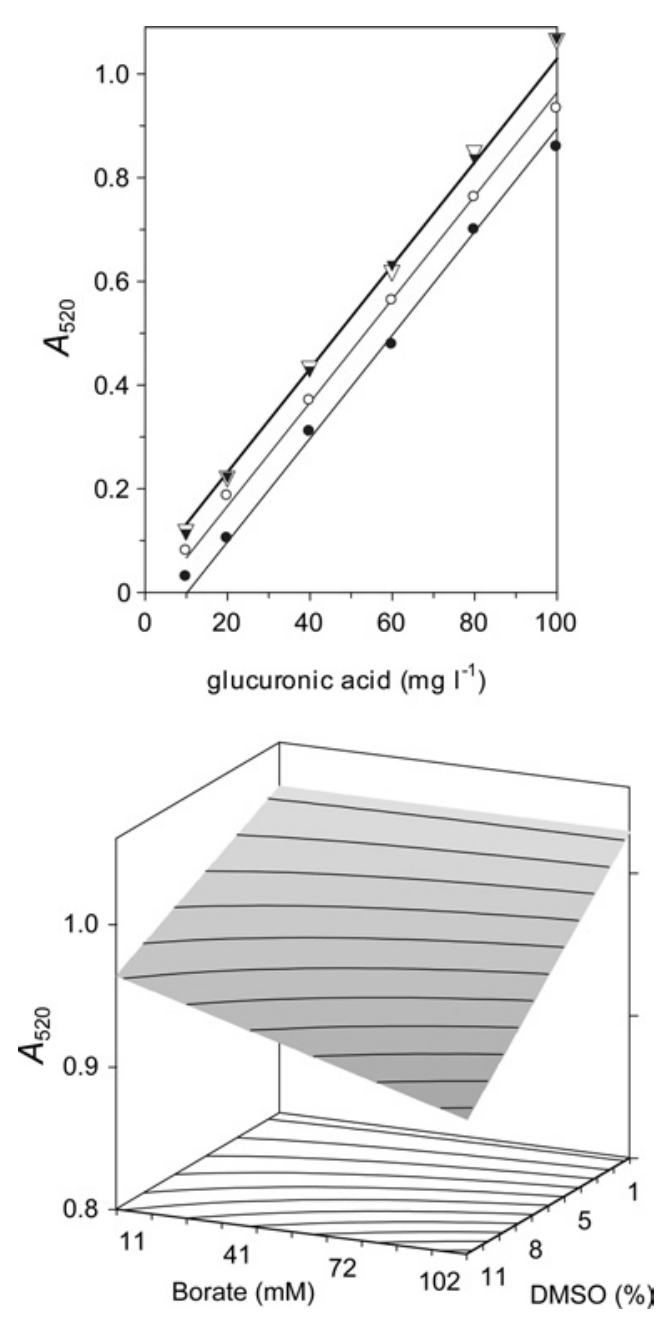

Figure I Combined effect of borate $\left({ }^{-} \mathrm{B}=0.85 \mathrm{mmol} \cdot \mathrm{I}^{-1} ;{ }^{+} \mathrm{B}=\right.$ $\left.102 \mathrm{mmol} \cdot \mathrm{I}^{-1}\right)$ and DMSO (-D $=0$; $\left.{ }^{+} \mathrm{D}=1 \mathrm{I} .4 \%\right)$ on the response of glucuronic acid

${ }^{-B^{-} D}(\nabla),{ }^{+} \mathrm{B}^{-} \mathrm{D}(\mathbf{\nabla}),{ }^{-} \mathrm{B}^{+} \mathrm{D}(\mathrm{O}),{ }^{+} \mathrm{B}^{+} \mathrm{D}(\mathbf{O})$. Lines correspond to the simultaneous fit of all series to eqn (I), whose surface response (for a concentration of $100 \mathrm{mg} \cdot \mathrm{ml}^{-1}$ glucuronic acid) is represented in the lower part of the Figure.

reaction mixture] of DMSO, the maximum of both reactants representing a situation equivalent to that of the microtitreplate assay. The results (Figure I) demonstrate that borate has no effect at the serial dilution studied and that DMSO depresses the response. In addition, the borate intensifies the effect of the DMSO. If $B$ is the borate concentration, $D$ the DMSO concentration and $U$ the glucuronic acid concentration, an empirical model of the type:

$$
Y=b_{0}+b_{\mathrm{u}} U-b_{\mathrm{d}} D-b_{\mathrm{db}} B D
$$

constitutes a satisfactory simultaneous solution $(r=0.9983)$ of the four series of responses. With $B$ in $\mathrm{mmol} \cdot \mathrm{I}^{-1}, D$ in $\%$

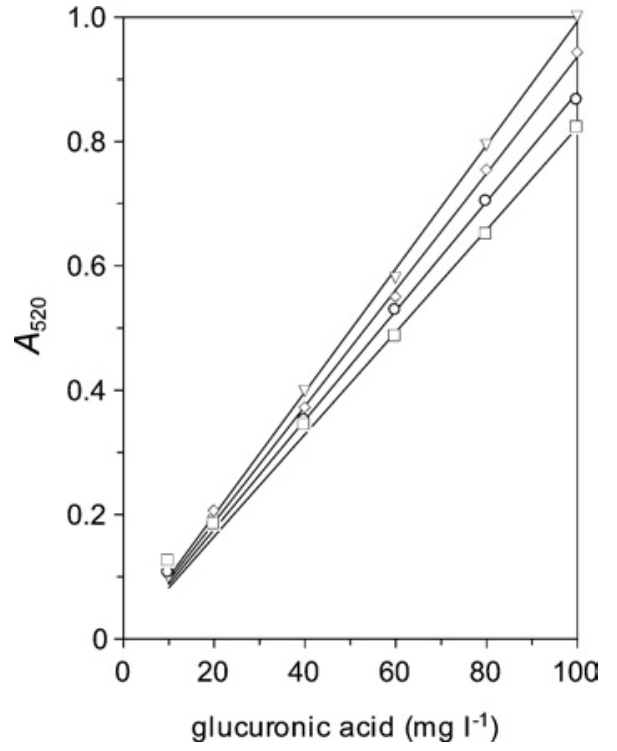

Figure 2 Response of increasing concentrations of pure glucuronic acid $(\square)$ in the presence of $5(\nabla), 10(\diamond)$ and $15(\bigcirc) \mathrm{g} \cdot \mathrm{I}^{-1}$ albumin

Points correspond to experimental values, lines to the simultaneous fit of all series to eqn (3b).

and $U$ in $\mathrm{mg} \cdot \mathrm{I}^{-1}$, the values of the coefficients are $b_{0}=0.033$; $b_{\mathrm{u}}=0.010 ; b_{\mathrm{d}}=0.006$ and $b_{\mathrm{db}}=0.00006$.

\section{Protein interference}

Figure 2 shows the response of the reaction of $m$-hydroxydiphenyl with a series of dilutions of glucuronic acid, alone and in the presence of three concentrations of albumin. Although interference is a small relative entity at the concentrations used, the results suggest that the protein plays a role in impeding the formation of the chromophore, either by reacting with the $m$-hydroxydiphenyl itself, thus blocking its reaction with uronic acid, or reacting with the uronic acid and blocking its combination with the reactant.

In view of the fact that both these possibilities are equivalent with regard to chromophore formation, we can limit the discussion to the first of them, assuming that the quantity of chromophore formed is a measure of its rate of formation. In the simplest hypothesis of a second-order reaction (first-order in each reagent), the chromophore formed (or $A$ measured, $Y$ ) can be expressed as a function of the concentrations of uronic acid $(U)$ and the available reactant $\left(R_{\mathrm{u}}\right)$, with $k_{\mathrm{u}}$ as a kinetic constant:

$$
Y=k_{\mathrm{u}} U R_{\mathrm{u}}
$$

The reactant available depends on the initial quantity $\left(R_{0}\right)$ and on that which is bound to the protein $\left(R_{\mathrm{p}}\right)$. Therefore, if $P$ is the protein concentration and $k_{\mathrm{p}}$ the corresponding kinetic constant, for reasons analogous to 

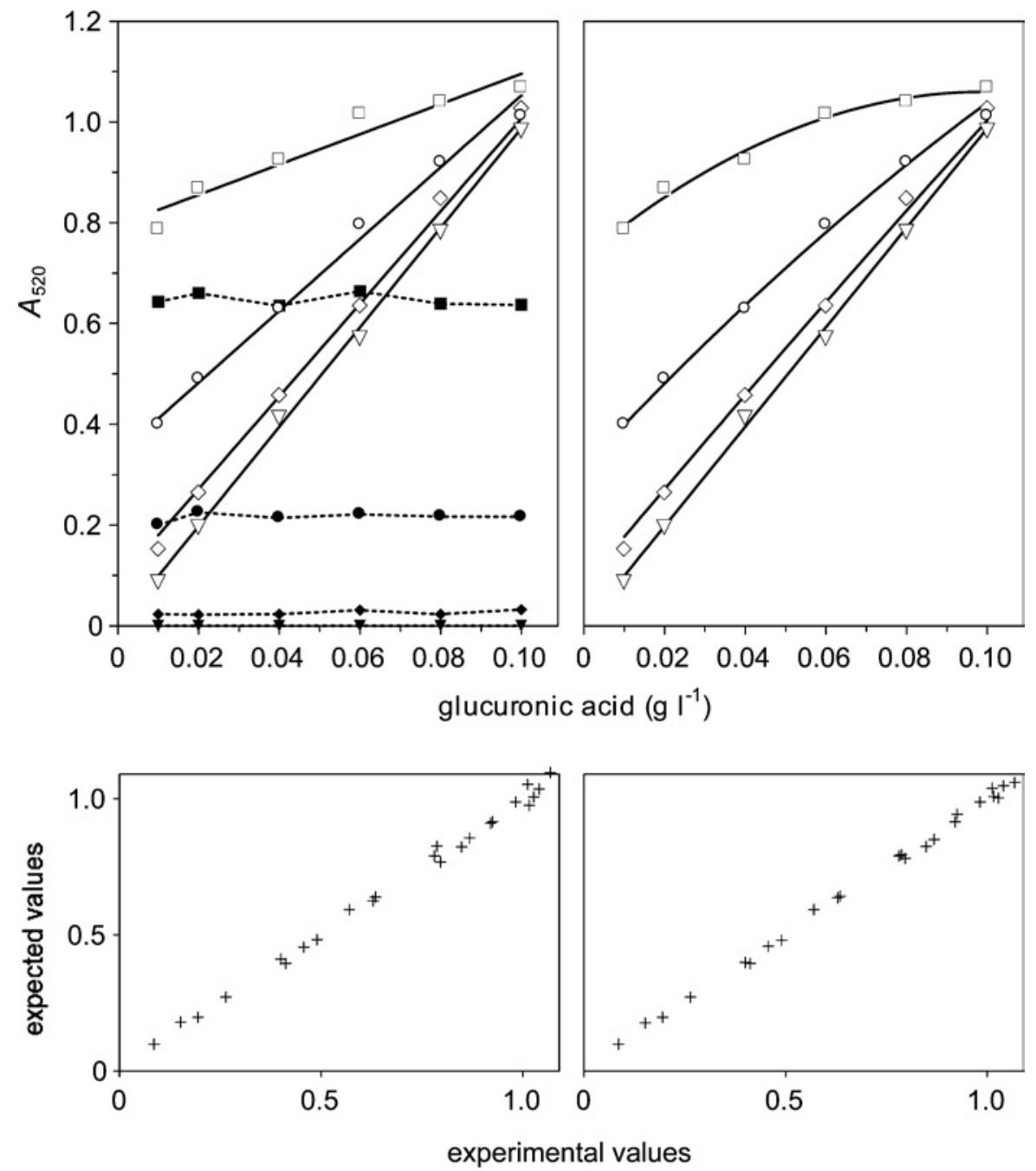

Figure 3 Response of increasing concentrations of pure glucuronic acid $(\boldsymbol{\nabla}, \nabla)$ in the presence of $0.25(\diamond, \diamond) I(\bullet, \bigcirc)$ and $2.5(\mathbb{\square}, \bigcirc) \mathrm{g} \cdot l^{-1}$ glucose, before (closed symbols) and after (open symbols) addition of $m$-hydroxydiphenyl (upper panels) and correlation between observed and model predicted values (lower panels)

In the upper panels, lines correspond to the simultaneous fit of all series of eqns (4a) (left-hand side) and (4b) (right-hand side).

those leading to eqn (2), the following can be formulated:

$$
R_{\mathrm{u}}=R_{0}-R_{\mathrm{p}} ; R_{\mathrm{p}}=k_{\mathrm{p}} P
$$

and therefore:

$$
Y=k_{\mathrm{u}} U\left(R_{0}-k_{\mathrm{p}} P\right)
$$

or, if $k_{\mathrm{u}} k_{\mathrm{p}}=k_{\mathrm{up}}$ :

$$
Y=k_{\mathrm{u}} R_{0} U-k_{\mathrm{up}} U P
$$

Assigning arbitrarily a value of $\mathrm{I}$ for $R_{0}$ and using eqn (3b) for the simultaneous description of the four series of Figure 2, the resulting minimum quadratic solution (quasiNewton method) is satisfactorily adjusted to the experimental values (correlation coefficient between observed and expected results, $r=0.9992$ ), obtaining $k_{u}=9.924$ and $k_{\mathrm{p}}=0.0 \mathrm{II}$ where $U$ and $P$ are expressed in $\mathrm{g} \cdot \mathrm{I}^{-1}$. It should be noted that although $k_{u}$ is much greater than $k_{p}$, in substrates such as vitreous humour the concentration of proteins can exceed that of the HA by two orders of magnitude.

When the experiment is completed by replacing glucuronic acid with a double concentration of purified $\mathrm{HA}$, the results are the same as those in Figure 2.

\section{Carbohydrate interference}

Carbohydrate interference, being more complex than for proteins, shows three types of effects.

(i) As shown in Figure 3, the presence of glucose increases the final response of the reaction. At least part of this contribution arises from the measured response after 
acid hydrolysis and before addition of the m-hydroxydiphenyl, and therefore only depends on the glucose present $(G)$, expressed as:

$$
Y_{1}=K_{g 1} G
$$

(ii) The effect of the glucose on the response cannot be expressed only as a function of the $Y$, value, corresponding to the measurement prior to addition of $m$-hydroxydiphenyl. Accordingly, a contribution depending on the reaction between glucose and the available reactant $\left(R_{g}\right)$ must be considered:

$$
Y_{2}=K_{g}^{\prime} G R_{g}
$$

where $R_{\mathrm{g}}=K^{\prime \prime}{ }_{\mathrm{g}} \mathrm{G}$; therefore:

$$
Y_{2}=K_{\mathrm{g} 2} G^{2}
$$

(iii) By accepting (ii), it is necessary to accept that there is competition for the reactant between glucuronic and glucose similar to that detected for proteins, although in this case glucose also contributes to the response. The effect can therefore be described by an equation formally identical with eqn ( $3 a)$, substituting the protein terms for analogous terms corresponding to glucose:

$$
Y_{3}=K_{\mathrm{u}} U\left(R_{0}-K_{g_{3}} G\right)
$$

Accordingly, the sum of the contributions $Y_{1}, Y_{2}$ and $Y_{3}$ produces the equation:

$$
Y=K_{g 1} G+K_{g 2} G^{2}+K_{u} U\left(R_{0}-K_{g 3} G\right)
$$

or, making $K_{\mathrm{u}} K_{\mathrm{g} 3}=K_{\mathrm{ug}}$,

$$
Y=K_{g 1} G+K_{g 2} G^{2}+K_{u} R_{0} U-K_{\mathrm{ug}} U G
$$

Again, if $R_{0}=\mathrm{I}$, the fit of the experimental results to this model, although acceptable (Figure 3, left-hand panels), notably improves by accepting that the simultaneous presence of glucose and glucuronic acid promotes a consumption of the reactants that is not simply additive. In this case, the contribution $Y_{3}$ is described as:

$$
Y_{3}=K_{\mathrm{u}} U\left[R_{0}-\left(K_{\mathrm{g} 3} G+K_{\mathrm{ug}} U G\right)\right]
$$

with a response, $Y$, of:

$$
Y=K_{\mathrm{g} 1} G+K_{\mathrm{g} 2} G^{2}+K_{\mathrm{u}} U\left[R_{0}-\left(K_{\mathrm{g} 3} G+K_{\mathrm{ug}} U G\right)\right]
$$

Combining homologous terms, making $R=\mathrm{I}$, and rearranging gives:

$$
Y=K_{g 1} G+K_{g 2} G^{2}+K_{u} U-K_{u g} U G-K_{u 2 g} U^{2} G
$$

\begin{tabular}{|c|c|c|c|c|c|}
\hline$U\left(\left.m g \cdot\right|^{-1}\right)$ & $P\left(\left.g \cdot\right|^{-1}\right)$ & $G\left(\left.g \cdot\right|^{-1}\right)$ & $A_{520}$ & $U_{\text {ap }}\left(\left.g \cdot\right|^{-1}\right)$ & $U_{\text {cor }}\left(\left.g \cdot\right|^{-1}\right)$ \\
\hline \multirow[t]{9}{*}{40} & 0 & 0 & 0.396 & 40.5 & 39.9 \\
\hline & & 0.25 & 0.458 & 46.7 & 39.8 \\
\hline & & 2.5 & 0.943 & 95.6 & 39.6 \\
\hline & 5 & 0 & 0.374 & 38.3 & 40.0 \\
\hline & & 0.25 & 0.436 & 44.5 & 39.9 \\
\hline & & 2.5 & 0.921 & 93.4 & 39.8 \\
\hline & 15 & 0 & 0.329 & 33.7 & 40.0 \\
\hline & & 0.25 & 0.391 & 40.0 & 39.9 \\
\hline & & 2.5 & 0.876 & 88.8 & 39.8 \\
\hline \multirow[t]{9}{*}{80} & 0 & 0 & 0.792 & 80.4 & 79.8 \\
\hline & & 0.25 & 0.824 & 63.6 & 79.6 \\
\hline & & 2.5 & 1.048 & 106.1 & 78.0 \\
\hline & 5 & 0 & 0.749 & 76.0 & 80.0 \\
\hline & & 0.25 & 0.781 & 79.3 & 79.9 \\
\hline & & 2.5 & 1.005 & 101.8 & 79.4 \\
\hline & 15 & 0 & 0.658 & 66.9 & 80.0 \\
\hline & & 0.25 & 0.690 & 70.1 & 79.8 \\
\hline & & 2.5 & 0.914 & 92.6 & 81.7 \\
\hline
\end{tabular}

whose minimum quadratic solution (quasi-Newton method) simultaneously describes the four experimental series of Figure 3, with a correlation coefficient between observed
Table I Apparent $\left(U_{\text {ap }}\right)$ and corrected $\left(U_{\text {cor }}\right)$ concentrations estimated from $A_{520}$ data obtained with two levels of glucuronic acid $(U)$ in the presence of the indicated concentrations of protein $(P)$ and glucose $(G)$

Corrected concentrations were obtained applying additively the models given by eqns (3b) and (4b).

and expected values of $r=0.9990$. With the concentrations of glucuronic acid and glucose in $\mathrm{g} \cdot \mathrm{I}^{-1}$, the values of the coefficients are $K_{\mathrm{g} 1}=0.329 ; K_{\mathrm{g} 2}=-0.015 ; K_{\mathrm{u}}=9.879$; $K_{\mathrm{ug}}=1.303$ and $K_{\mathrm{u} 2 \mathrm{~g}}=13.352$. The results are unchanged when glucuronic acid is replaced with a double concentration of purified HA.

\section{Absence of interactions between proteins and carbohydrates}

As described in the Materials and methods section, the effect of the simultaneous presence of proteins and carbohydrates was studied by means of a rotatable design [II,I2] appropriate to validate an equation of the type:

$$
Y=b_{0}+b_{1} P+b_{2} G+b_{12} P G+b_{11} P^{2}+b_{22} G^{2}
$$

When the results were adjusted to such an equation, the lack of statistical significance for coefficients $b_{12}$ (interaction protein-glucose) and $b_{11}$ (quadratic effect of the protein) was found. Therefore, it can be concluded that the effects of proteins and glucose are simply additive and they can be independently corrected as a function of the concentrations present in a concrete biological matrix. Even an equation like eqn (5), once the non-significant coefficients are eliminated and the remaining ones recalculated, could be used for the correction of the interferences. It is clear, however, that the model given by eqn (5) represents an excessively simplified version of the sum of eqns (3b) and (4b), thus resulting in less precise corrections. The best alternative is to apply eqns (3b) and (4b), as is shown in Table I. 


\section{Kinetic (differential) approach}

Despite the previous sections containing a hypothesis relative to reaction rates, the validating data were concentrations of reactants and products after a determined reaction time. In addition, the models proposed (especially in the case of the carbohydrates) included terms of reasonable physical significance, but derived from purely empirical arguments. Although the practical use of these models for the correction of interference has been demonstrated, it seems of interest to show that those (integral) equations proposed are coherent with those that can be deduced from (differential) kinetic considerations. On the other hand, although the variation of the response with time was not studied, the results of the experimental measures at the end of the reaction time are also predictable by means of the differential models.

\section{Proteins}

Two parallel second-order reactions are implied: one between $\mathrm{R}$ (reactant) and $\mathrm{P}$ (protein), and another between $R$ and $U$ (uronic acid):

$$
R+U \stackrel{q_{u}}{\longrightarrow} Y
$$

where $R_{0}, U_{0}$ are the concentrations at $t=0$, and $\left(R_{0}-Y\right)$, $\left(U_{0}-Y\right)$ at $t=t$.

$$
R+P \stackrel{q_{\mathrm{p}}}{\longrightarrow} X
$$

where $R_{0}, P_{0}$ are the concentrations at $t=0$, and $\left(R_{0}-X\right)$, $\left(P_{0}-X\right)$ at $t=t$.

Under these conditions, the colour formed will be $Y_{\mathrm{w}}=Y-X$, with a formation rate of:

$$
\mathrm{d} Y_{\mathrm{w}} / \mathrm{d} t=\mathrm{d} / \mathrm{d} t(Y-X)=\mathrm{d} Y / \mathrm{d} t-\mathrm{d} X / \mathrm{d} t
$$

where:

$$
\mathrm{d} Y / \mathrm{d} t=q_{\mathrm{u}}\left(R_{0}-Y\right)\left(U_{0}-Y\right)
$$

and:

$$
\mathrm{d} X / \mathrm{d} t=q_{\mathrm{p}}\left(R_{0}-X\right)\left(P_{0}-X\right)
$$

In the first equation, $Y^{\prime}=f(Y, t)$, separating variables and integrating, one obtains:

$$
\int_{0}^{y} \frac{d Y}{\left(R_{0}-Y\right)\left(U_{0}-Y\right)}=\int_{0}^{t} q_{u} d t
$$

whose solution leads to the following explicit expression of Y:

\begin{tabular}{|c|c|c|c|}
\hline \multirow[b]{2}{*}{ Parameter } & \multirow[b]{2}{*}{ Approach ... } & \multicolumn{2}{|l|}{ Value $\left(1 \cdot \mathrm{g}^{-1} \cdot \mathrm{min}^{-1}\right)$} \\
\hline & & Empirical & Kinetic \\
\hline $\begin{array}{l}k_{u} \text { and } q_{u} \\
k_{u p} \text { and } q_{p}\end{array}$ & & $\begin{array}{l}k_{u}(\text { eqn } 3 b)=0.0331 \\
k_{\text {up }}(\text { eqn } 3 b)= \\
3.64 \times 10^{-4}\end{array}$ & $\begin{array}{l}q_{u}(\text { eqn } 6)=0.0456 \\
q_{p}(\text { eqn } 7)= \\
1.97 \times 10^{-5}\end{array}$ \\
\hline$K_{u}$ and $Q_{u}$ & & $K_{u}($ eqn $4 b)=0.0324$ & $Q_{u}($ eqn 10$)=0.0363$ \\
\hline
\end{tabular}

$$
Y=\frac{R_{0} U_{0}\left(\mathrm{e}^{q_{\mathrm{u}}\left(R_{0}-U_{0}\right) t}-\mathrm{I}\right)}{R_{0} \mathrm{e}^{q_{\mathrm{u}}\left(R_{0}-U_{0}\right) t}-U_{0}}
$$

The second equation, $X^{\prime}=f(X, t)$, of analogous solution,
Table 2 Comparison between the parametric estimations obtained, in the different models, by means of the two approaches indicated

leads to:

$$
X=\frac{R_{0} P_{0}\left(\mathrm{e}^{q_{p}\left(R_{0}-P_{0}\right) t}-\mathrm{I}\right)}{R_{0} \mathrm{e}^{q_{p}\left(R_{0}-P_{0}\right) t}-P_{0}}
$$

therefore, finally, the value of $Y_{w}$ will be:

$$
\begin{aligned}
Y_{q}= & Y-X=\frac{R_{0} U_{0}\left(\mathrm{e}^{q_{\mathrm{u}}\left(R_{0}-U_{0}\right) t}-\mathrm{I}\right)}{R_{0} \mathrm{e}^{q_{\mathrm{u}}\left(R_{0}-U_{0}\right) t}-U_{0}} \\
& -\frac{R_{0} P_{0}\left(\mathrm{e}^{q_{\mathrm{p}}\left(R_{0}-P_{0}\right) t}-\mathrm{I}\right)}{R_{0} \mathrm{e}^{q_{\mathrm{p}}\left(R_{0}-P_{0}\right) t}-P_{0}}
\end{aligned}
$$

To determine the values of the coefficients $q_{u}$ and $q_{p}$ it should be noted that when $P_{0}=0$ the second term in eqn (8) is cancelled, leaving $Y_{\mathrm{w}}=Y$, thus deriving $q_{\mathrm{u}}$ :

$$
q_{\mathrm{u}}=\frac{\mathrm{I}}{\left(R_{0}-U_{0}\right) t} \ln \left[\frac{U_{0}\left(R_{0}-Y_{\mathrm{w}}\right)}{R_{0}\left(U_{0}-Y_{\mathrm{w}}\right)}\right]
$$

whose numerical value, substituted into eqn (8), allow the calculation of $q_{p}$.

Accordingly, it is now necessary to obtain the numerical values of $q_{u}$ and $q_{p}$ from eqn (8), and check their compatibility with those calculated for the coefficients $k_{\mathrm{u}}$ and $k_{\text {up }}$ in eqn (3b). The mean value of $q_{u}$ can be obtained from an experimental series of $P_{0}=0$, making $R_{0}=I$, inserting the reaction time $(t=15 \mathrm{~min})$, optic length $(L=\mathrm{I} \mathrm{cm})$ and absorption coefficient of the chromophore $(\varepsilon)$. In practice, however, the exact value of $\varepsilon$ can be ignored, since it is also present in the conversion necessary for expression of $k_{\mathrm{u}}$ $\left(\mathrm{I}^{2} \cdot \mathrm{g}^{-2}\right)$ and $q_{\mathrm{u}}\left(\mathrm{I} \cdot \mathrm{g}^{-1} \cdot \mathrm{min}^{-1}\right)$ in the same units:

$$
\begin{aligned}
q_{\mathrm{u}}\left(\mathrm{l} \cdot \mathrm{g}^{-1} \cdot \mathrm{min}^{-1}\right)= & k_{\mathrm{u}}\left(l^{2} \cdot \mathrm{g}^{-2}\right) /\left[\varepsilon\left(\mathrm{l} \cdot \mathrm{g}^{-1} \cdot \mathrm{cm}^{-1}\right)\right. \\
& \cdot L(\mathrm{~cm}) \cdot t(\mathrm{~min})]
\end{aligned}
$$

Under these conditions an arbitrary value can be used for $\varepsilon$, provided that it is identical in eqns (8) and (10) and the argument of the Naperian (natural) logarithm (In) in eqn (9) is positive. The values thus obtained for $q_{u}$ and $k_{u}$ (both in $1 \cdot \mathrm{g}^{-1} \cdot \mathrm{min}^{-1}$ ) show good agreement (Table 2 ). An analogous treatment is applicable to the coefficients $q_{p}$ in eqn (8) and $k_{\text {up }}$ in eqn (3b), the values of which are also given in Table 2. 


\section{Carbohydrates}

Maintaining the usual notations for glucose $(G)$, uronic $(U)$, reactant $(\mathrm{R})$ and colour formed $(\mathrm{Y})$, this case implies the following parallel reactions.

(I) Conversion (first-order) of glucose, independent of the reactant

$$
G \stackrel{Q_{g 1}}{\longrightarrow} Y_{1}
$$

where $G_{0}$ is the concentration at $t=0$, and $\left(G_{0}-Y_{1}\right)$ at $t=t$.

(2) Reaction (second-order) between glucose and reactant

$$
R+G \stackrel{Q_{g 2}}{\longrightarrow} Y_{2}
$$

where $R_{0}, G_{0}$ are the concentrations at $t=0$, and $\left(R_{0}-Y_{2}\right)$, $\left(G_{0}-Y_{2}\right)$ at $t=t$.

(3) Reaction (second-order) between uronic acid and reactant

$$
R+U \stackrel{Q_{u l}}{\longrightarrow} Y_{3}
$$

where $R_{0}, U_{0}$ are the concentrations at $t=0$, and $\left(R_{0}-Y_{3}\right)$, $\left(U_{0}-Y_{3}\right)$ at $t=t$.

(4) Reaction (second-order) between glucose and uronic acid

$$
G+U \stackrel{Q_{\mathrm{U} 2}}{\longrightarrow} Y_{4}
$$

where $G_{0}, U_{0}$ are the concentrations at $t=0$, and $\left(G_{0}-Y_{4}\right)$, $\left(U_{0}-Y_{4}\right)$ at $t=t$. Therefore, the colour formed is:

$$
Y_{w}=Y_{1}+Y_{2}+Y_{3}+Y_{4}
$$

and rate of formation:

$$
\begin{aligned}
\mathrm{d}_{\mathrm{w}} / \mathrm{d} t & =\mathrm{d} / \mathrm{d} t\left(Y_{1}+Y_{2}+Y_{3}+Y_{4}\right) \\
& =\mathrm{d} Y_{1} / \mathrm{d} t+\mathrm{dY}_{2} / \mathrm{d} t+\mathrm{d}_{3} / \mathrm{d} t+\mathrm{d}_{4} / \mathrm{d} t
\end{aligned}
$$

where:

$$
\begin{aligned}
& \mathrm{d} Y_{1} / \mathrm{d} t=Q_{\mathrm{gl}}\left(G_{0}-Y_{1}\right) ; \\
& \mathrm{d} Y_{2} / \mathrm{d} t=Q_{\mathrm{g} 2}\left(R_{0}-Y_{2}\right)\left(G_{0}-Y_{2}\right) ; \\
& \mathrm{d} Y_{3} / \mathrm{d} t=Q_{\mathrm{ul}}\left(R_{0}-Y_{3}\right)\left(U_{0}-Y_{3}\right) \\
& \mathrm{d} Y_{4} / \mathrm{d} t=Q_{\mathrm{u} 2}\left(G_{0}-Y_{4}\right)\left(U_{0}-Y_{4}\right)
\end{aligned}
$$

As for proteins, integration of these equations between the limits $t=0, Y=0$ and $t=t, Y=Y$, gives:

$$
\begin{aligned}
Y_{w}= & G_{0}\left(I-\mathrm{e}^{-Q_{g 1} t}\right)+\frac{R_{0} G_{0}\left(\mathrm{e}^{Q_{g 2}\left(R_{0}-G_{0}\right) t}-\mathrm{I}\right)}{R_{0} \mathrm{e}^{Q_{g 2}\left(R_{0}-G_{0}\right) t}-G_{0}} \\
& +\frac{R_{0} U_{0}\left(\mathrm{e}^{Q_{\mathrm{u} 1}\left(R_{0}-U_{0}\right) t}-\mathrm{I}\right)}{R_{0} \mathrm{e}^{Q_{\mathrm{ul}}\left(R_{0}-U_{0}\right) t}-U_{0}} \\
& +\frac{G_{0} U_{0}\left(\mathrm{e}^{Q_{\mathrm{u} 2}\left(G_{0}-U_{0}\right) t}-\mathrm{I}\right)}{G_{0} \mathrm{e}^{\mathrm{e}_{\mathrm{u} 2}\left(G_{0}-U_{0}\right) t}-U_{0}}
\end{aligned}
$$

Therefore, when $G_{0}=0, Y_{\mathrm{w}}=Y_{3}$, the solution for $Q_{\mathrm{ul}}$ is:

$$
Q_{\mathrm{ul}}=\frac{\mathrm{I}}{\left(R_{0}-U_{0}\right) t} \ln \left[\frac{U_{0}\left(R_{0}-Y_{\mathrm{w}}\right)}{R_{0}\left(U_{0}-Y_{\mathrm{w}}\right)}\right]
$$

As in the previous case, the numerical value for $Q_{u l}$ can be obtained from the results of an experimental series with $G_{0}=0$, and comparing (Table 2) with results calculated for the coefficient $K_{u}$ of eqn (4a), expressed in the same units as $Q_{u l}$ by means of an analogous transformation to eqn (10). In contrast with the case of protein; however, it is not possible to compare the remaining coefficients of eqns (4a) and (II), since they cannot be isolated in eqn (II).

It should be noted that the agreement between both approximations is satisfactory in all the cases where comparison is possible, which supports our hypothesis. Undoubtedly, the kinetic approximation for carbohydrates corresponds to the model described by eqn (4a) and not eqn (4b), in spite of the better fit of the experimental results. This can be attributed to the fact that the interaction in eqn (4b) has various possible kinetic translations, and it would be speculative to postulate a concrete argument in absence of reaction mechanism data.

\section{An example}

Let us suppose a sample with $A_{520}=0.930$, containing $5 \mathrm{~g} \cdot \mathrm{I}^{-1}$ albumin, $2.5 \mathrm{~g} \cdot \mathrm{l}^{-1}$ glucose and an unknown concentration of glucuronic acid $(U)$. To determine $U$, we have the equations that describe the following.

(a) The response of pure glucuronic acid $(U)$, i.e., a calibration curve

$$
Y_{U}=c_{0}+c_{1} U=-0.0053+9.92 U\left(c_{0} \text { can be rejected }\right)
$$

(b) The joint response of $U$ and protein $(P)$ interference

$$
Y_{\mathrm{UP}}=k_{\mathrm{u}} U-k_{\mathrm{u}} k_{\mathrm{p}} U \mathrm{P}\left(\text { note } R_{0}=\mathrm{I} \text { and } k_{\mathrm{up}}=k_{\mathrm{u}} k_{\mathrm{p}}\right. \text { ) }
$$

where $k_{\mathrm{u}}=9.924$ and $k_{\mathrm{p}}=0.01 \mathrm{I}$

(c) The joint response of $U$ and glucose $(G)$ interference

$$
Y_{\mathrm{UG}}=K_{\mathrm{g} 1} G+K_{g^{2}} G^{2}+K_{\mathrm{u}} U-K_{\mathrm{ug}} U G-K_{\mathrm{u} 2 g} U^{2} G
$$

where $K_{\mathrm{g} 1}=0.329, K_{\mathrm{g} 2}=-0.015, K_{\mathrm{u}}=9.879, K_{\mathrm{ug}}=1.303$ and $K_{\mathrm{u} 2 \mathrm{~g}}=13.352$.

Then, if we make $P=0$ (absence of proteins) in $Y_{\text {UP }}$, it gives:

$$
Y_{\mathrm{UPO}}=k_{\mathrm{u}} U
$$

which represents the response due to $U$. This way the difference $Y_{U P}-Y_{U P O}$ represents the response specifically due 
to $P$ in the presence of $U$ :

$$
Y_{\mathrm{P}}=Y_{\mathrm{UP}}-Y_{\mathrm{UPO}}=-k_{\mathrm{u}} k_{\mathrm{P}} U P
$$

Applying the same reasoning (with $G=0$ ) in $Y_{U G}$, we obtain the difference, $Y_{U G}-Y_{U G 0}$, that represents the response specifically due to $\mathrm{G}$ in the presence of $\mathrm{U}$ :

$$
Y_{\mathrm{G}}=Y_{\mathrm{UG}}-Y_{\mathrm{UG} 0}=K_{\mathrm{g} 1} G+K_{\mathrm{g} 2} G^{2}-K_{\mathrm{ug}} U G-K_{\mathrm{u} 2 \mathrm{~g}} U^{2} G
$$

Since there are no interactions, the joint response of $U, P$ and $G$ (the experimental value $A_{520}=0.930$ ) can be written:

$$
\begin{aligned}
0.930= & Y_{\mathrm{U}}+Y_{\mathrm{P}}+Y_{\mathrm{G}}=9.92 U-k_{\mathrm{u}} k_{\mathrm{p}} U P \\
& +K_{\mathrm{g} 1} G+K_{\mathrm{g} 2} G^{2}-K_{\mathrm{ug}} U G-K_{\mathrm{u} 2 \mathrm{~g}} U^{2} G
\end{aligned}
$$

an expression in which we know the numeric values of $P$, $G$ and all the parameters. Rearranging it with $U$ as sole unknown, we obtain a quadratic equation in which:

$$
\begin{aligned}
& -\left(k_{\mathrm{u}} k_{\mathrm{p}} P+K_{\mathrm{ug}} G-9.92\right) \pm \\
& \frac{\left[\left(k_{\mathrm{u}} k_{\mathrm{p}} P+K_{\mathrm{ug}} G-9.92\right)^{2}\right.}{\left.-4 K_{\mathrm{u} 2 \mathrm{~g}} G\left(0.930-K_{\mathrm{g} 1} G-K_{\mathrm{g} 2} G^{2}\right)\right]^{1 / 2}}
\end{aligned}
$$

By substituting $P, G$ and parameters for their numeric values, the two possible solutions are:

$$
\begin{aligned}
& U_{1}=0.140 \mathrm{~g} \cdot \mathrm{I}^{-1} \text { or } \\
& U_{2}=0.042 \mathrm{~g} \cdot \mathrm{I}^{-1} \text { (without correction, } 0.094 \mathrm{~g} \cdot \mathrm{I}^{-1} \text { ) }
\end{aligned}
$$

The decision is immediate. Since we know the concentrations of proteins and glucose, as well as the characteristics of their effects, the feasible value will be necessarily lower that the apparent one (the one obtained without correction), i.e. $0.042 \mathrm{~g} \cdot \mathrm{l}^{-1}$. Naturally, if $P=0$ or $G=0$, eqn $(A)$ takes the forms:

$$
\begin{aligned}
P= & 0 \rightarrow 0.930=Y_{U}+Y_{G}=9.92 U \\
& +K_{g 1} G+K_{g 2} G^{2}-K_{u g} U G-K_{u 2 g} U^{2} G
\end{aligned}
$$

and

$$
G=0 \rightarrow 0.930=Y_{\mathrm{U}}+Y_{\mathrm{P}}=9.92 U-k_{\mathrm{u}} k_{\mathrm{p}} U P
$$

It should be pointed out that the description of the proposed method is much more prolix than its practical application, this being reducible to a trivial calculation routine on a spreadsheet. The coefficients of these equations are applicable to the correction of interference due to glucose and albumin, the most habitual species in the biological materials that contain $\mathrm{HA}$, and the determination of which is a routine in the context of the methods for obtaining $\mathrm{HA}$, as well as in clinical analysis. Other sugars and other proteins could produce other values in the coefficients (gelatin interference can be solved with values equal to those obtained for albumin), easily calculable from experimental data and maintaining the forms of the equations proposed. On the other hand, the precedent example (as well as Table I) shows the utility of the resource, since in many biological materials the error can even exceed $200 \%$ if the analysis is carried out without the suggested correction.

\section{References}

I Toyoda, H., Motoki, K., Tanikawa, M., Shinomiya, K., Akiyama, H. and Imanari, T. (|99|) J. Chromatogr. 565, I4I-|48

2 Tulamo, R. M. (199|) Am. J. Vet. Res. 52, 1940-1944

3 Hong, M., Sudor, J., Stefansson, M. and Novotny, M. V. (1998) Anal. Chem. 70, 568-573

4 Blumenkrantz, N. and Asboe-Hansen, G. (1973) Anal. Biochem. 54, 484-489

5 Van den Hoogen, B. M., Van Weeren, P. R., Lopes-Cardozo, M., Van Golde, L. M. G., Barneveld, A. and Van de Lest, C. H. A. (1998) Anal. Biochem. 257, 107-I। I

6 Thibault, J. F. (1979) Lebensm. Wiss. Technol. 12, 247-25।

7 Filisetti-Cozzi, T. M. and Carpita, N. C. (199|) Anal. Biochem. 197, $157-162$

8 Cullis-Hill, D. (1989) U.S. Patent 4,879,375

9 Lowry, O. H., Rosebrough, N. J., Farr, A. L. and Randall, R. J. (I95I) J. Biol. Chem. 193, 265-275

I0 Rodén, L., Baker, J. R., Cifonelli, J. A. and Mathews, M. B. (1972) Methods Enzymol. 28B, 73-140

I I Box, G. E. P., Hunter, W. G. and Hunter, J. S. (1978) Statistics for Experimenters, John Wiley and Sons, New York

12 Akhnazarova, S. and Kafarov, V. (1982) Experiment Optimization in Chemistry and Chemical Engineering, MIR, Moscow

Received 5 August 2004; accepted 27 August 2004

Published as Immediate Publication 27 August 2004, DOI 10.I042/BA20040I 27 